\title{
Public Health Burden of Botulinum Neurotoxicity: A Research Perspective to Evaluate the Antitoxin Efficacy of Thearubigin Extract of Black Tea
}

\author{
Rifat Nawaz Ul Islam ${ }^{1}$ and Subhasree Ray ${ }^{2 *}$ \\ (Department of Biotechnology, University of Burdwan, India) \\ (Department of Food Science \& Nutrition, SNDT Women's University, India)
}

\begin{abstract}
Botulinum toxin (BoNT), being the most poisonous substance poses a great threat to global public health as a deadly bio-weapon. BoNT/A, BoNT/B and BoNT/E serotype toxicity cause flaccid paralysis in human by blocking neurotransmitter activity. Thearubigin, a black tea extract is found to be effective in neutralizing BoNT when experimented with animal models. This hypothesis opens a new frontier of Biological Science to understand the antitoxin property of thearubigin and applying the same in large scale to prevent BoNT neurotoxicity. Individualized dosage recommendation of thearubigin extract thus represents a natural antitoxin therapy in managing severe botulism in humans.
\end{abstract}

Keywords: Bio-Weapon, BoNT/A, BoNT/B, BoNT/E, Metal Chelating, Public Health, SNARE Protein, Thearubigin

\section{Introduction}

Botulism, a rare but life threatening, mainly food borne disease caused by a toxic bacterial protein Botulinum (BoNT) ${ }^{1}$, well known neurotoxin expressed in an anaerobic spore forming bacteria Clostridium botulinum. Human neurotoxin BoNT elicits flaccid paralysis by a very unique mechanism which includes the entrance of BoNT into the body, survival of the toxin in the cytosol and neurotransmitter blocking activity by applying metalloendopeptidase \& catalytic property of zinc as a whole ${ }^{1}$. Hemagglutinin (HA) protein, an adjuvant for BoNT vaccine is a carrier \& protector of BoNT in cytosol environment which help the toxin in crossing the cell barriers ${ }^{2}$. HA protein even cannot be isolated from BoNT complex in vaccine production due to this strong association ${ }^{2}$. Though the neurotoxin BoNT have seven serotypes termed BoNT/A through BoNT/G; BoNT/A, BoNT/B, BoNT/E and rarely BoNT/F play major role in human neurotoxicity ${ }^{1}$ and ultimately BoNT/B and BoNT/A, BoNT/Es successfully block neurotransmitter release by breaking a tight complex of Soluble N-ethylmaleimide-Sensitive Factor(NSF)Attachment Protein REceptor (SNARE) proteins bridge ${ }^{3}$, connecting to synaptic vesicle and plasma membranes by chopping out a small C-terminal peptide fragment of vesicle-associated membrane protein 2(VAMP2) or synaptobrevin present in synaptic vesicle and synaptosomalassociated protein of $25 \mathrm{kDa}$ (SNAP25) present in plasma membrane respectively ${ }^{3}$. Due to this fatal neurological action, botulinum toxin is a potent bio-weapon for spreading terrorism and poses an immense threat on public health. In this context the perspective study aims to discuss the mechanism of botulinum toxicity and possible intervention strategy to manage the same by taking preventive as well as curative aspect of health into consideration. Proper food processing with optimum $\mathrm{pH}$, temperature and water activity is always an important part of preventing microbial contamination of food. So, to prevent botulinum toxicity food preservation should be taken as one of the most crucial steps in respect to public health disease management. Along with prevention, to stop toxicity in humans, antioxidant property of nutritional flavonoids can be applied. Nutritional science research has shown that black tea extract, thearubigin is effective in blocking botulinum neurotoxicity ${ }^{4}$. Within the scope of Nutritional biochemistry and computational Biology, it can be established that BoNT is effectively blocked by thearubigin when applied in proper dosage. Even, this research is of utmost importance as common vaccine preparation is a challenge due to the difference in mechanism of action for different botulinum serotypes.

We have thus tried to explain some broad aspects of BoNT toxicity and possible nutritional intervention in this perspective study -

1. Botulinum toxin as a potent public health threat and powerful bio-weapon.

2. Mechanism of action of BoNT/A \& BoNT/E and BoNT/B in causing flaccid paralysis in human.

3. Sound food processing and preservation as one of the most crucial steps in preventing BoNT toxicity.

4. Possible mechanism of black tea extract in blocking BoNT neurotoxicity and practical implication with the help of Computational Biology and Nutritional Biochemistry.

5. Future perspective of managing BoNT in respect of global public health. 


\section{Botulinum, A Threat To Public Health And A Powerful Bioweapon}

Botulism is a rare disease with four naturally occurring syndromes, food-borne botulism is caused by ingestion of foods contaminated with botulinum toxin, wound botulism is caused by clostridium botulinum colonization in the wound and in situ toxin production, infant botulism is caused by intestinal colonization and toxin production and adult intestinal toxemia botulism is an even rarer form of intestinal colonization and toxin production in adults ${ }^{1}$. All forms of botulism produce the same distinct clinical syndrome of symmetrical cranial nerve paralysis of voluntary muscles, which may progress to respiratory comprise and death. Globally, botulism is fairly rare. Between 1990 and 2000, the Centres for Disease Control reported 263 individual cases of food borne botulism ${ }^{5}$.

Botulinum toxin poses a major bio weapon threat because of its extreme potency and lethality, its ease of production, transport \& misuse and the need for prolonged intensive care among affected population. An outbreak of botulism contributes a medical emergency that requires prompt provision of botulinum antitoxin and constitutes a medical emergency that requires immediate intervention to prevent additional cases. Botulinum toxin is the most poisonous substances known. A single gram of crystalline toxin, evenly dispersed and inhaled would kill more than one million people. The basis of the phenomenal potency of botulinum toxin is enzymatic; the toxin is a zinc proteinase that cleaves one or more fusion proteins by which neural vesicles release acetylcholine into the neuromuscular junction ${ }^{3}$.

\subsubsection{History of Botulinum Toxin As a Bio-Weapon:}

Botulinum is the first biological toxin that has licensed for treatment of human diseases. In United States, botulinum toxin is currently licensed for treatment of cervical torticollis, strabismus and blepharospasm associated with dystonia. It is also used 'off label' for a variety of more prevalent conditions that include migraine headache, chronic low back pain, stroke, traumatic brain injury, cerebral palsy, achalasia and various dystonias.

Terrorists have already attempted to use botulinum toxin as a bio-weapon. Aerosols are dispersed at multiple sites in Downtown in Tokyo, Japan and at US military installations in Japan on at least three occasions between 1990 and 1995 by the Japanese cult ${ }^{6}$. The perpetrators obtained their C.botulinum from soil that they had collected in Northern Japan ${ }^{6}$. The US biological weapons program first produced botulinum toxin during World War II ${ }^{6}$.

\section{Mechanism Of Action Of BoNT}

In human body, toxicity of botulinsm is occurred by a toxic metaloprotein with the help of botulinum complex proteins. BoNT produced as progenitor toxins of large molecular sizes (Figure - 1) of 19S (LL toxin); $12 \mathrm{~S}$ (M toxin) consists of a nontoxic, non-hemagglutinin component (NTNHA) and the neurotoxin (BoNT); 16S ( $\mathrm{L}$ toxin) consists of the $\mathrm{M}$ toxin and the 3 subcomponents of hemagglutinin (HA) ${ }^{2}$. HA is composed of subcomponents having 70,33 , and $17 \mathrm{kDa}$. The $70 \mathrm{kDa}$ subcomponent undergoes proteolytic processing and is split into HA-55 and HA-22-232.

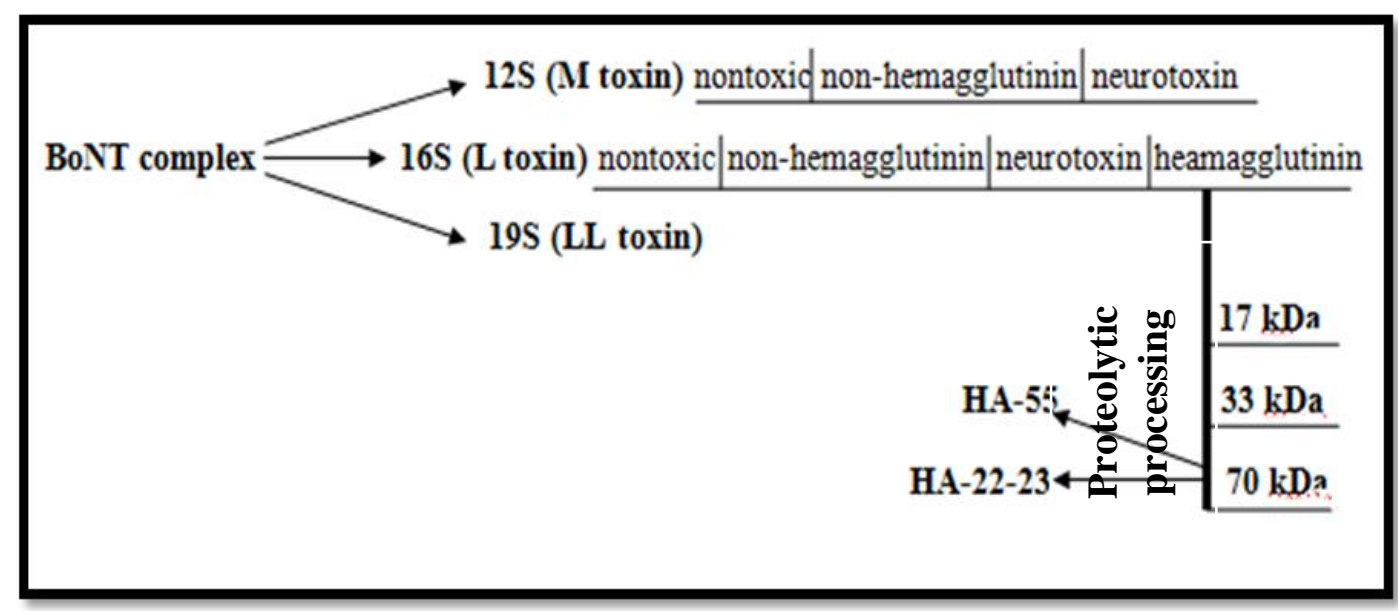

Figure - 1: BoNT complex proteins

Botulinum toxicity is a long term and serotype independent process. BoNT toxin travels a long distance that comprises a pathway of mouth to stomach to gut to epithelial membrane and finally to synaptic membrane or vesicle ${ }^{3}$. 


\subsection{Mode of Action of BoNT A and BoNT $E$}

BoNT/A \& BoNT/E have near about same mode of action with an exception in cleaving the synaptoprotein in the vesicles. The process of the toxicity is describes in the following section -

\subsubsection{Transcytosis from stomach to intestine}

From mouth BoNT enters into the acidic environment of stomach with an ease and the high acidity of stomach is capable of conformational change in BoNT/A \& BoNT/E. To be protected from high acidic condition and enzymatic digestion, BoNT become covered by a special type of non-toxic HA protein called HA33 or $\mathrm{HA}^{2}$. HA complex of serotype BoNT/A, BoNT/E bound to the cell adhesion protein E-cadherin ${ }^{7}$. The HA complex recognizes E-cadherin and also binds to carbohydrates on the cell surface. ${ }^{7}$. Binding of the HA complex sequesters E-cadherin in the monomeric state, compromising the E-cadherin-mediated intercellular barrier and facilitating paracellular absorption of BoNT/A, BoNT/E. After transporting into the intestine, the alkaline $\mathrm{pH}$ of intestine separates BoNT from associated proteins, HA-33. The naked BoNT is then absorbed via intestinal epithelial or $\mathrm{M}$ cells $\mathrm{s}^{3,7}$

\subsubsection{Entrance to blood from intestine}

Then BoNT/A, /E is attaches with NTNHA and three types of HA proteins; 3 HA-70/HA3, 6 HA33/HA-34/HA1, 3 HA-17/HA2. This progenitor BoNT protein complex easily carry BoNT/A, /E to blood by the help of a combination of HA-33, HA-17 \& HA-55 proteins (HA-55 is derived from HA-70 by a proteolytic process, another subunit is HA-22-23) as these combination of protein is necessary for hemagglutination activity $^{3}$.

\subsubsection{Removal of HA}

After crossing epithelial cell barrier BoNT/A, /E become uncoated dimer by releasing HA and NTNHA proteins in the blood ${ }^{8}$.

\subsubsection{BoNT/A, /E binding to SV2 A, B and ganglioside GT1B}

BoNT/A, /E then get attached with Synaptic vesicle glycoproteins SV2A or SV2B or SV2C and ganglioside protein GT1B. In vivo, this process specifically targets synapses at neuromuscular junctions, where toxin association with ganglioside may position it to bind efficiently to SV2A, SV2B, or SV2C when those proteins are exposed at the cell surface by exocytose ${ }^{9}$.

\subsubsection{Generation of LC fragment}

Synaptic vesicles re-formed rapidly after exocytosis, carrying vesicle membrane proteins that had been exposed on the cell surface by exocytosis back into the cell. The botulinum toxin type A disulfide-bonded heavy chain - light chain dimer (BoNT/A HC:LC) bound to ganglioside GT1b and synaptic vesicle protein 2A, 2B, or $2 \mathrm{C}(\mathrm{SV} 2 \mathrm{~A}, \mathrm{~B}$, or $\mathrm{C})$ is inferred to be taken up as well, delivering it to the re-formed synaptic vesicle ${ }^{10}$.

\subsubsection{Enzymatic LC fragment transportation to neuron cytosol}

Due to recycling of synaptic vesicle, acidification occurs and BoNT/, /E undergo conformational changes by breaking disulfide bond between heavy chain and light chain of these serotypes. Then BoNT/A, /E: LC: $\mathrm{Zn}^{+}$become enzymatically functional and moves to neuron cytosol. This movement is mediated by the help of GT1B. BoNT/A,/E:HC goes back to plasma membrane through recycling process ${ }^{10}$.

\subsubsection{Catalytic activity of $\mathrm{Zn}^{2+}$}

Then the pathway leads to entrance of BoNT/A,/E: $\mathrm{LC}_{\mathrm{Zn}}{ }^{+}$to target cell SNAP25 (Synaptosomalassociated protein 25 ) and catalyzed by $\mathrm{Zn}^{+}$ion. A water molecule at $250^{\text {th }}$ position of SNAP25 is converted into a nucleophile which is held by one or two $\mathrm{Zn}^{+}$ion(s). Water containing oxygen molecule itself is highly electronegative and have four pairs of electrons where two pairs are neutralized by two Hydrogen molecules, another one pair interacts with $\mathrm{Zn}^{+}$ion and then to get stabilized the remaining pair of electron is released which hydrolyses charged amino acid of SNAP25 by binding covalently with $\mathrm{C} \alpha$ of peptide bond at particular position $^{2,10}$. 


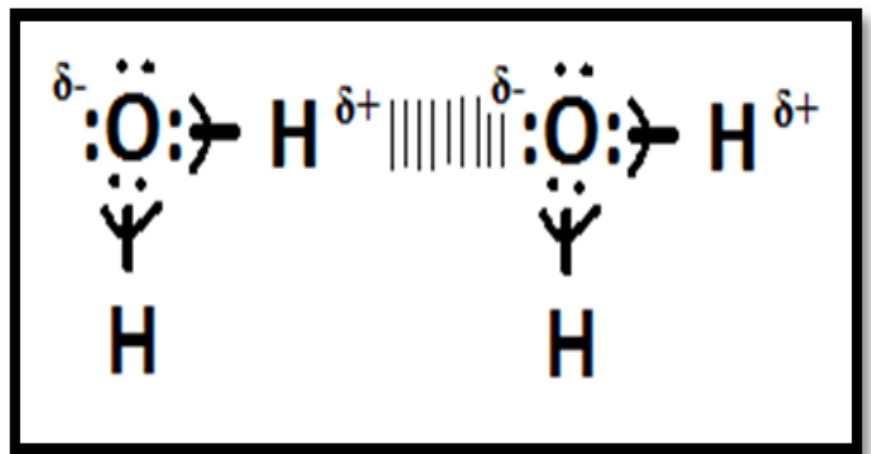

Figure - 2: Nucleophile property of water for highly electronegative Oxygen.

\subsubsection{Cleavage on SNAP25}

BoNT/A: LC: $\mathrm{Zn}+$ \& BoNT/E: LC: $\mathrm{Zn}^{+}$hydrolyses the $\mathrm{C} \alpha$ peptide bond of Glutamic acid (Glu) at position $197^{\text {th }}$ and Arginine (Arg) at $198^{\text {th }}$ position of SNAP25 respectively. As a result SNAP25 becomes unable to connect with Synaptobrevin or syntaxin ${ }^{2,10}$ (shows in figure - 3).
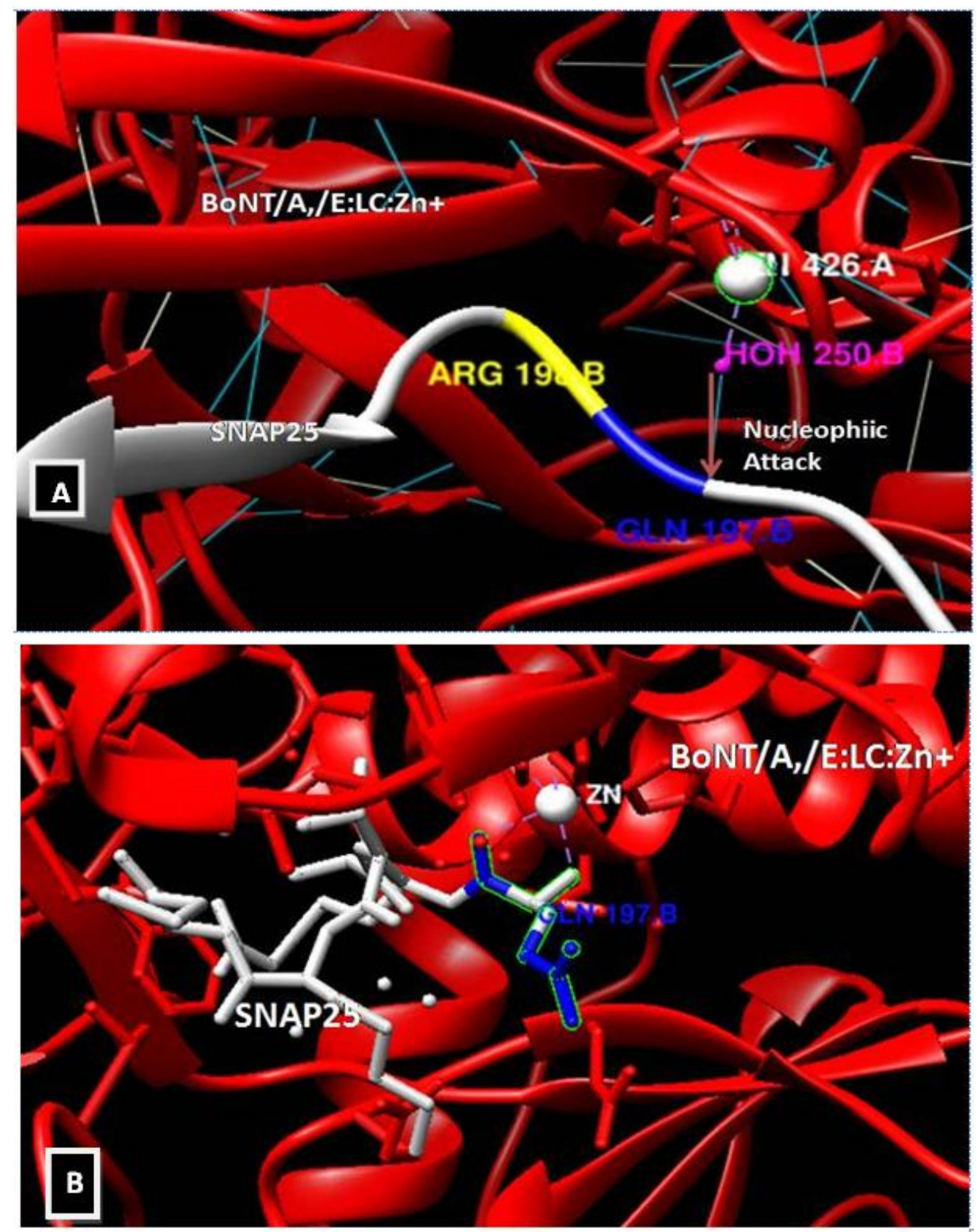

Figure -3: White color protein is SNAP25and red one is toxic BoNT/A, /E : LC: $\mathrm{Zn+}+(\mathrm{A})$ shows neucleophilic attacking position before cleavage and (B) shows copped SNAP25 C-terminal peptide. 


\subsubsection{Blockage of neurotransmitter}

Ultimately neurotransmitter activity is blocked to receive or exert any message to the brain that causes flaccid paralysis.

\subsection{Mode of Action of BoNT/B}

The mechanism of action of BoNT/B is almost same as serotype A \& E to some extent before it enters the target cell. BoNT/B target cell is vesicle-associated membrane protein 2 (VAMP2). The mechanism of BoNT/B toxicity is as follows -

\subsubsection{BoNT/B binding to SYT 1,2 and ganglioside GT1B}

BoNT/B binds to ganglioside GT1B and syntagmin 1 or 2 (SYT 1, 2) of Synaptotagmin vesicle present in human plasma membrane. This ganglioside GT1B helps BoNT/B to efficiently bind with SYT 1 or 2 when those proteins are exposed at the cell surface by exocytosis ${ }^{11}$.

\subsubsection{Generation of LC fragment}

Synaptic vesicles reformed rapidly after exocytosis and carrying exposed vesicle membrane proteins SYT 1, 2 back into the cell. The botulinum toxin type B disulfide-bonded heavy chain - light chain dimer (BoNT/B HC: LC) binds to ganglioside GT1b and syntagmin 1 or 2 (SYT 1,2) is inferred to be taken up as well, delivering it to the re-formed synaptic vesicle ${ }^{11}$.

\subsubsection{Enzymatic LC fragment transport to neuron cytosol}

Acidification during recycling of synaptic vesicle causes conformational changes and beaks down disulfide bond between HC and LC of BoNT/B. Subsequently GT1B helps BoNT/B: LC to act as an enzyme and extruded into the neuronal cytosol. BoNT/B: HC part goes back by recycling procedure ${ }^{11}$.

\subsubsection{Catalytic activity of $\mathrm{Zn}^{2+}$}

After getting enzymatic property BoNT/B:LC: $\mathrm{Zn}^{2+}$ goes to VAMP2 and with the help of $\mathrm{Zn}^{2+}$ nucleophile water molecule it cleaves VAMP2 into two fragments. This cleavage is occurred in $\mathrm{C} \alpha$ peptide bond at glutamate (Gln) 76 in VAMP2 ${ }^{11}$ (shows in figure -4).

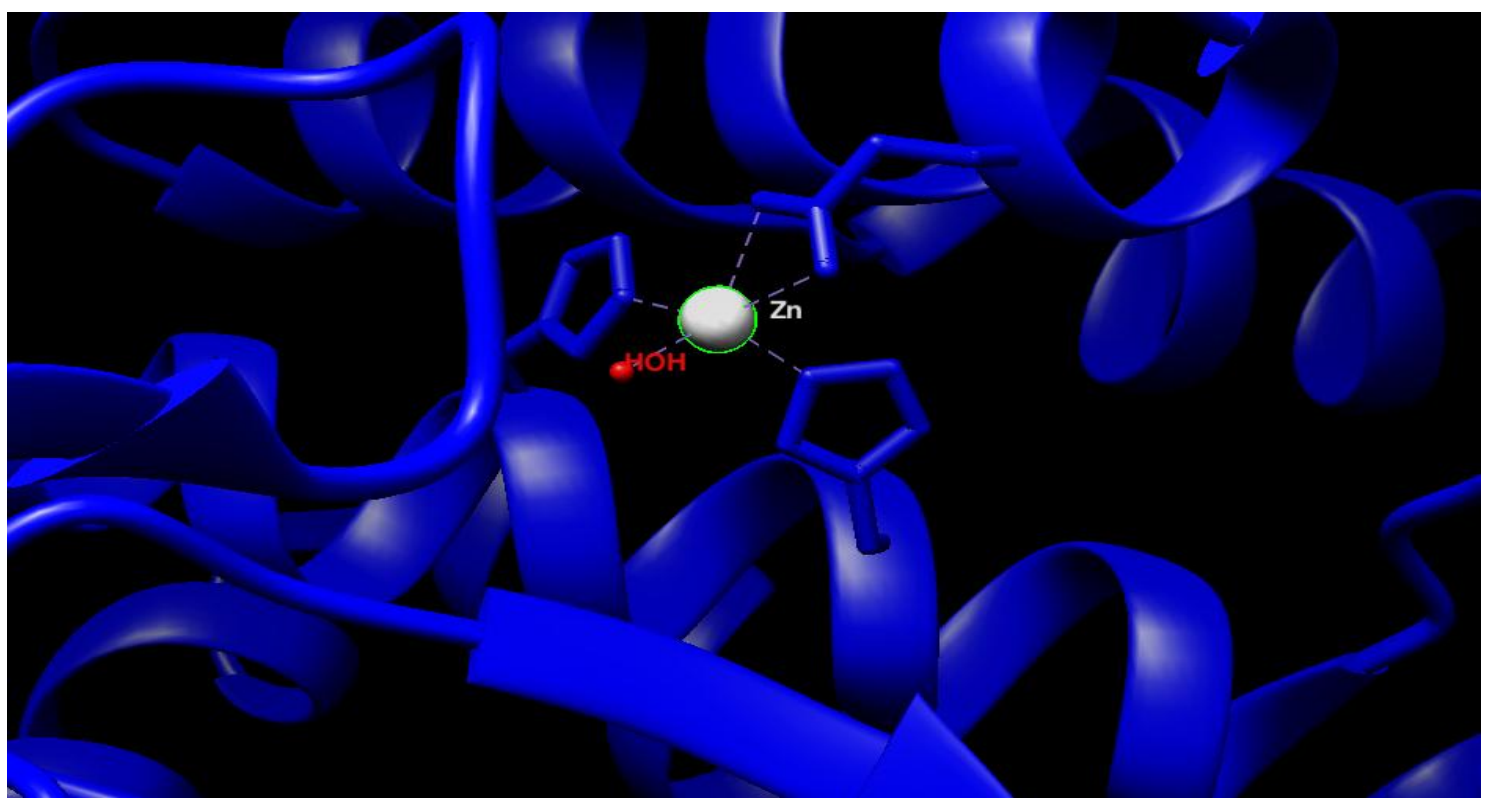

Figure-4: BoNT/B active site containing Zn and a neucleophilic Oxygen

\subsubsection{Blockage of neurotransmitter}

VAMP2 $\mathrm{C}$ terminal returns to cytosol and $\mathrm{N}$ terminal remains in vesicle that finally leads to blocking of neurotransmitter ${ }^{11}$.

\section{Management Of Botulinum Toxin:- Preventive Aspect}

C.botulinum is present in soil and water; so, potentially any food that comes into contact with such vectors is a potential hazard. Home canned products, especially low acid food products, are attributed to most cases of food borne botulinum as low $\mathrm{pH}(<4.5)$ is favourable for growth of the spore. Foods commonly 
associated with botulism are canned asparagus, green beans, garlic in oil, corn, soups, ripe olives, tuna fish, sausage, luncheon meats, fermented meats, salad dressings, and smoked fish. Spores have also been found on the surfaces of vegetables and fruits. Infant botulism has been linked to the ingestion of C.botulinum spores in honey, corn syrup, and other foods. During the canning process, foods undergo a hot fill process and oxygen is removed, leaving the food in an anaerobic environment. Certain foods, such as meat, are able to bind oxygen to create an anaerobic environment that allows C.botulinum to grow. Home canning processes for low acid foods are extremely risky because the time and temperature food are heated are often inadequate. On a commercial scale, improperly handled food products have also contributed to outbreaks ${ }^{12}$.

\subsection{Preventive Strategy by Safe Processing, Preservation and Handling of Food}

The main limiting growth factors for $\mathrm{C}$. botulinum are extreme temperature, $\mathrm{pH}$ below 4.6 , low $\mathrm{a}_{\mathrm{w}}$, food preservatives and additives. Proper cooking and handling are important in reducing the number of C. botulinum spores in the food. While most bacteria cannot survive at a low $\mathrm{pH}$, some proteins such as in soy and beef have protective agents that allow them to grow at $\mathrm{pH}<4.5$. Low water activity inhibits the growth of $\mathrm{C}$. botulinum, which is why dehydrated foods and foods high in salt and/or sugar do not support its growth. Food preservatives such as nitrites, sorbic acid, phenolic antioxidants, polyphosphates, and ascorbates, as well as lactic acid bacteria, inhibit the growth of C. botulinum ${ }^{12}$.

Most outbreaks of foodborne botulism are the result of poor home canning. Proper time, temperature, and pressure are required to destroy the heat-resistant spores, and proper storage methods are necessary to ensure the safety of the consumer. A pressure cooker can be used for home canning purposes because it can reach temperatures higher than boiling $\left(212^{\circ} \mathrm{F}\right)$, which is necessary to kill the spores ${ }^{12}$.

While the botulinum spores can survive in boiling water, the botulinum toxin is heat-labile. Heating food to a typical cooking temperature of $80^{\circ} \mathrm{C}\left(176^{\circ} \mathrm{F}\right)$ for 10 minutes before consumption can greatly reduce the risk of illness.

The suggestions below are good examples of how to prevent foodborne C. botulinum toxicity ${ }^{12}$ :

- If consuming home canned foods, heat low acid foods to at least $80^{\circ} \mathrm{C}\left(176^{\circ} \mathrm{F}\right)$ for 10 minutes and corn, spinach, and meats for 20 minutes.

- Oils infused with garlic or herbs should be properly refrigerated.

- Canned food products, both home and commercial, should be inspected before use. Cans with bulging or damaged lids, leakage, or off odors should not be used because growth of the bacteria can often produce a gas, causing the can to expand.

- Home canned foods should be canned in pressure cookers to ensure the proper time, temperature, and pressure requirements to avoid the growth of the bacteria and spores.

\section{Possible Intervention With Black Tea Extract:-Practical Implementation With} Nutritional Biochemistry And Computational Biology

Nutritional Biochemistry and food toxicology in their scope of research is continuously investigating to develop food compatible conditions to alter the structures of fungal, bacterial and plant toxins, thus transforming toxins to non-toxic molecules. Animal studies have shown that black tea extract, thearubigin has potentially stopped the botulinum neurotoxicity when added to the toxin. There are series of experimental studies those demonstrated that thearubigin fraction when added to BoNT/A, BoNT/E and BoNT/B has effectively blocked the neurotoxicity in mice and prevented death. These studies open a new frontier in Medical studies where dosage of thearubigin can be formed, implemented and use in large scale in protecting botulinum neurotoxicity. The dosage can be formulated and measured in individual basis with the help of Computational Biology.

Thearubigin are complex polyphenols which are formed during fermentation by polymerization of theaflavins, during the manufacture of black tea from the enzymatic oxidation of cathechins by polyphenol oxidase ${ }^{4}$. Thearubigin, inhibits free radical generations, inactivates prooxidative enzymes and chelate transition metal ions $^{4}$. Although the structure of thearubigin has not been completely clarified, it is a compound having the structure of the following chemical formula - 
<smiles>O=c1c(O)cc([C@@H]2Oc3cc(O)cc(O)c3C[C@H]2O)cc(C(CCO)[C@@H]2C[C@@H](O)Cc3c(O)cc(O)cc3O2)c1O</smiles>

Figure - 5: Thearubigin molecule (Wherein $\mathrm{R}$ is a galloyl group) ${ }^{4}$

\subsection{Why Thearubigin?}

C. botulinum loses its toxicity only after a long period of treatment at high temperature, which may not be applicable for some types of food. Further it prefers anaerobic conditions found in preserved foods such as bottled and canned products making its prevention even more difficult. On the other hand, chemical neutralizing agents and disinfectants which may be highly effective against botulinum toxin but also harmful to human body. Functionally BoNT/A, BoNT/E and BoNT/B are different in causing neurotoxicity and a common vaccine can't be recommended for prevention as described in the mechanism in action. In this context thearubigin can be used as an antioxidant-antitoxin food molecule in blocking the BoNT toxin. The perspective study assumes two possible hypotheses of thearubigin mechanism as an antitoxin. One common property of botulinum serotypes includes presence of $\mathrm{Zn}^{2+}$ which can be chelated by thearubigin and resulting into neutralizing the toxin. Thearubigin can also act in scavenging the highly negative electron from nucleophilic water molecule, produced by $\mathrm{Zn}^{2+}$ to prevent cleavage of Glu and Arg in the SNAP25 protein.

\subsection{Practical Implication}

Existing invention demonstrated that thearubigin is effective in neutralizing the neurotoxin production by C.botulinum and C.tetani. The neutralization is probably based on inhibition, reduction and annihilation of any negative function of the toxins produced by these micro-organisms. The perspective study tries to add some new researchable hypotheses in existing knowledge of food toxin management study. Thearubigin, a natural extract of tea is harmless to humans and environment thus can be utilized in therapeutic purpose as an antitoxin. It can be used to detoxify BoNT as first line of defense while food processing and preservation. The extract can be used in cleaning wounds infected with botulinum toxin by spreading, spraying or wiping. Further, it can be used as medicine to treat botulinum toxicity in infected population.

\section{Future Aspect And Challenges}

The current knowledge of existing research study approves that black tea weakens the neurotoxin of C.botulinum, and thearubigin, the brown coloured pigment of black tea has function in neutralizing the toxin produced by the microorganism. This invention is of immense importance to establish thearubigin extract as a powerful neutralizer of BoNT. Nutritional Biochemistry, Computational Biology and Biotechnology altogether can be applied in producing a purified recommended dose of thearubigin for medicinal use. The challenges are to understand the interaction of thearubigin with BoNT and assessing possible side effects of thearubigin before it is used for pharmaceutical purpose in toxin neutralization.

\section{Discussion}

The seven serotypes of botulinum toxin produced by C.botulinum exert their paralytic effect by inhibiting acetylcholine release at the neuromuscular junction. Each of these zinc endopeptidases cleaves one or more proteins involved in vesicle transport and membrane fusion. Among these seven serotypes, BoNT A, BoNT B and BoNT E are most dangerous for humans. BoNT has reported as the most poisonous substances and poses a great threat to public health. Even, BoNT has used as a bio-weapon during past warfare. This severity of botulinum toxin is of immense importance in the field of Biology and thus a new paradigm has been shifted into establishing thearubigin as a treatment by understanding the mechanism of neutralization of the toxin by recommending personalized dosage.

\section{Conclusion}

The perspective study is a combination of literature that explains severity of botulinum toxin as a potent bio-weapon and neurotransmitter blocker that poses a great threat on global public health. The toxin is 
known to be most toxic substance and kill a population within a second if it is inhaled. This increases application of botulinum toxin in terrorist activities and Biological sciences in their scope of expertise continuously experimenting in developing a treatment for this toxin. Serotype A, B and E are specifically dangerous for human in causing flaccid paralysis and shows different mechanism of action and this limits in forming a common vaccine for all serotypes. In this context, black tea polyphenol, thearubigin has been demonstrated to block botulinum toxin in several animal studies. The studies also explained the mechanism of anti-toxicity of thearubigin with several possible assumptions. The perspective study tries to provide some distinct hypotheses to understand the mechanism of thearubigin anti-toxicity to accelerate the process of food technological research in dosage formulation and practical implication of black tea extract in the field of 'Medical Nutrition Therapy' to prevent botulinum toxicity in global population.

\section{References}

[1] P. K. Nigam and A. Nigam, Botulinum toxin, Indian J Dermatol, 55(1), 2010, 8-14.

[2] S. Ali, E. Firouz, H. Abbas, R. Mosayeb and K. Hani, Expression and Purification of Neurotoxin-Associated Protein HA-33/A from Clostridium botulinum and Evaluation of Its Antigenicity, Iran Biomed J, 17(4), 2013, 165-170.

[3] D. Ungar and F. M. Hughson, SNARE protein structure and function, Annu Rev Cell Dev Biol, 19, $2003,493-517$.

[4] E. Satoh, T. Ishii, Y. Shimizu, S. Sawamura and M. Nishimura, The mechanism underlying the protective effect of the thearubigin fraction of black tea (Camellia sinensis) extract against the neuromuscular blocking action of botulinum neurotoxins, Pharmacol Toxicol, 90(4), 2002, 199-202.

[5] C. Mazuet, E. Ezan, H. Volland, M. R. Popoff and F. Becher, Toxin detection in patients' sera by mass spectrometry during two outbreaks of type A Botulism in France, J Clin Microbiol,50(12), 2012, 4091-4.

[6] P. Bossi and F. Bricaire, Botulism toxin, bioterrorist weapon, Presse Med, 32(10), 2003, 463-5.

[7] K. Lee, X. Zhong, S. Gu, A. M Kruel, M. B Dorner, K. Perry et al., Molecular basis for disruption of Ecadherin adhesion by botulinum neurotoxin A complex, Science, 344(6190), 2014, 1405-10.

[8] S. Miyashita, K. Niwa, T. Watanabe and Y. Sagane, Host-cell specificity and transcytosis of nontoxic nonhemagglutinin protein of botulinum neurotoxin serotype D, FEMS Microbiol Lett, 357(2), 2014, 115-22.

[9] A. R. Kroken, A.P. Karalewitz, Z. Fu, J. J. Kim and J.T. Barbieri, Novel ganglioside-mediated entry of botulinum neurotoxin serotype D into neurons, J Biol Chem, 286(30), 2011, 26828-37.

[10] K. Lee, S. Gu, L. Jin, T. T. Le, L. W. Cheng, J. Strotmeier et al., Structure of a bimodular botulinum neurotoxin complex provides insights into its oral toxicity, PLoS Pathog, 9(10), 2013, e1003690.

[11] A. Potulska-Chromik, B. Zakrzewska-Pniewska, E. Szmidt-Sałkowska, J. Lewandowski, M. Siński, W. Przyjałkowski et al., Long lasting dysautonomia due to botulinum toxin B poisoning: clinical-laboratory follow up and difficulties in initial diagnosis, BMC Res Notes, 6, 2013, 438 .

[12] Centers for Disease Control and Prevention. 1998. Botulism in the United States, 1899-1996. Handbook for epidemiologists, clinicians, and laboratory workers. Atlanta, GA: U.S. Department of Health and Human Services, CDC. Available from :http://www.cdc.gov/ncidod/dbmd/diseaseinfo/files/botulism.pdf. Accessed August 11, 2014. 